\title{
Interaction potentials from periodic density- functional theory calculations: Molecular- dynamics simulations of Au clusters deposited on the TiN (001) surface
}

Cite as: J. Chem. Phys. 123, 244706 (2005); https://doi.org/10.1063/1.2140702

Submitted: 21 June 2005 . Accepted: 01 November 2005. Published Online: 28 December 2005

N. Cruz Hernández, and Javier Fdez. Sanz

ARTICLES YOU MAY BE INTERESTED IN

A climbing image nudged elastic band method for finding saddle points and minimum energy paths

The Journal of Chemical Physics 113, 9901 (2000); https://doi.org/10.1063/1.1329672

TiC and TiN supported platinum monolayer as high-performance catalysts for CO oxidation: A DFT study

The Journal of Chemical Physics 149, 054705 (2018); https://doi.org/10.1063/1.5038857

\section{Lock-in Amplifiers up to $600 \mathrm{MHz}$}
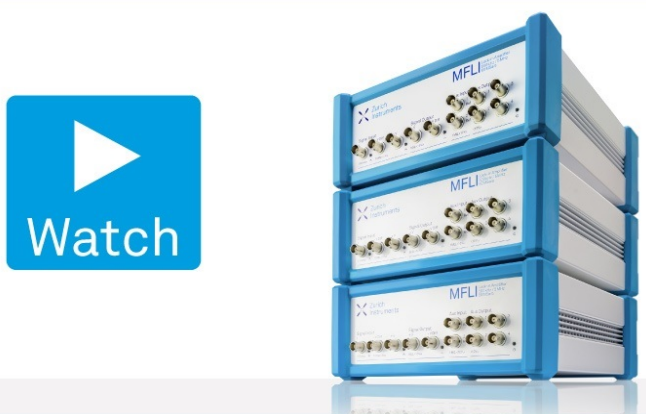

J. Chem. Phys. 123, 244706 (2005); https://doi.org/10.1063/1.2140702 


\title{
Interaction potentials from periodic density-functional theory calculations: Molecular-dynamics simulations of Au clusters deposited on the TiN (001) surface
}

\author{
N. Cruz Hernández and Javier Fdez. Sanz ${ }^{\text {a) }}$ \\ Departamento de Química Física, Facultad de Química, Universidad de Sevilla, E-41012 Sevilla, Spain
}

(Received 21 June 2005; accepted 1 November 2005; published online 28 December 2005)

\begin{abstract}
Molecular-dynamics simulations of gold particles deposited on a TiN (001) surface have been accounted for through classical pair potentials describing the atom force field. The interaction between $\mathrm{Ti}-\mathrm{N}, \mathrm{Ti}-\mathrm{Ti}, \mathrm{N}-\mathrm{N}, \mathrm{Au}-\mathrm{Au}, \mathrm{Au}-\mathrm{Ti}$, and $\mathrm{Au}-\mathrm{N}$ pairs was estimated by following a procedure in which the interaction energy between two sets of atoms is estimated from density-functional calculations performed with periodic boundary conditions using plane waves as basis set. The pair potentials were expressed as the sum of two contributions: long range in a Coulomb form and a short-range term, which included the rest of the energy contributions. Simulations of the TiN (001) isolated surface reproduced the already described surface relaxation, with a rippling parameter in agreement with that found from a purely first-principles approach. Simulations of gold deposition on such surfaces showed the formation of metal clusters with well-defined fcc structure and epitaxially grown. (C) 2005 American Institute of Physics. [DOI: 10.1063/1.2140702]
\end{abstract}

\section{INTRODUCTION}

Density-functional theory, ${ }^{1}$ using plane-wave functions and periodic boundary conditions (p-DFT), constitutes one of the most powerful tools nowadays in computer material science (see Ref. 2 for a review). Within the p-DFT framework, static properties of medium-sized systems (about 100 atoms) are currently being simulated. However, in spite of the brilliant elegance of this methodology, its application to large systems is restricted by the high computational cost. Therefore, the study of problems with relatively large models must be undertaken using other kinds of methods. An additional aspect to consider concerns the study of the dynamic behavior of a system. For instance, $a b$ initio moleculardynamics (aiMD) simulations ${ }^{3}$ based on the DFT calculations constitute an extraordinarily powerful tool in this field. Recently, computational power has increased to the point where the aiMD methods based on the p-DFT calculations can be used to describe many processes of chemical interest and may be a suitable option if the interest is in systems of relatively small dimensions. With this method it is possible to take into account the changes of the electronic structure of the system during the simulation. However, morphology of three-dimensional metal particles on the surface can be obtained only if we use models of larger dimensions.

Classical molecular-dynamics (cMD) simulations ${ }^{4}$ constitute one of the most effective methodological alternatives for the study of problems that require models with large numbers of particles. With this approach it is possible to use models with thousands of particles. However, in the cMD methods the correct election of the force field that defines the

\footnotetext{
${ }^{\text {a) }}$ Author to whom correspondence should be addressed. Electronic mail: sanz@us.es
}

interaction between atoms has long been an important bottleneck in the theoretical modeling of many types of physical systems.

Intermolecular and interatomic interaction potentials (force fields) are used in a vast area of modern science. In the simplest cases, i.e., for interactions between atoms and small linear molecules, the potentials are usually computed on a first-principles basis or fitted to experimental data. Assuming a global functional for the system, a full potential-energy surface (PES) can be obtained by fitting the data or by interpolating in some manner between the known data points. Therefore, once a set of potential energies is computed on a grid of points, an analytic function has to be fitted to these data in order to be used in various types of simulations. Many forms of such functions have been proposed in literature but a consensus as to which of them is most effective is yet to be determined. Along with this, the fact that currently available accurate analytical fits of finite discrete sets of $a b$ initio potential-energy data typically involve large numbers of adjustable parameters. To avoid any requirement of chemical intuition as well as the possibility of unphysical features being introduced, an alternative procedure makes use of interpolation methods that do not need a mathematical expression to represent the interaction between atoms. In this case the potential surface is just a set of data on a dense grid and the actual values of the potential are estimated from, for instance, spline interpolation.

One of the most common ways to represent the interaction in a group of atoms is by using pairwise interaction potentials. According to this, the sum of all pair interactions results in a global PES formulation. Such interaction potentials may take varied forms and usefully represented as a sum of a long-range Coulomb interaction and short-range interaction. In most cases the short-range contribution includes parameters that are fit in order to agree with the experimental 
results or ab initio calculations. However, independent of the expression that has been used to describe the short-range interaction potential, a more practical procedure to perform the simulations is to build up a table of values of the potential and forces with respect to the square distance. Then, the contributions to the energy and forces are obtained by numerical interpolation. This alternative reduces the computational cost of the simulations but can also be used to set up interaction potential that does not match a given analytical expression.

The interaction potentials used in this work follow this approach: instead of looking for an expression to analytically fit the potential, the interaction between two species is estimated in a range (dense) of interatomic distances and directly used in the simulations. With this aim, the short-range potential contribution is estimated in this work from periodic DFT calculations using a recently outlined ${ }^{5}$ mathematical procedure that will be fully described in Sec. II. In this procedure the interaction energy between a given set of particles is represented by means of a collection of vectors and coefficients obtained by solving a system of linear equations built up using the DFT energies obtained in a close dense range of interatomic distances. A simplified variant to this method that used a diagonal approach to the system of equations was also employed to simulate the behavior of $\mathrm{Pd}_{64}$ clusters deposited on a clean $\alpha-\mathrm{Al}_{2} \mathrm{O}_{3}$ (0001) surface. ${ }^{6}$ Both the morphology of the transition-metal particle and the stability with respect to the temperature were found to agree with the experimental data.

The procedure to obtain interaction pair potentials, Sec. III, will be subsequently used to analyze the structural properties of Au clusters deposited on titanium nitride (001) surface by means of the MD simulations. Titanium nitride, TiN, is a material of high interesting technological and catalytic applications because of its exceptional physical and chemical properties, which make it attractive both from fundamental and experimental points of view. ${ }^{7,8}$ Transition-metal nitrides are commonly referred to as refractory metals as they have extreme hardness and a high melting point, properties comparable to those of covalent crystals, as well as good corrosion resistance and metallic conductivity close to the reported values of pure transition metals. TiN has also attracted interest as a support for the deposition of transition-metal clusters, both for engineering purposes (sensors, electronic devices, etc.,) and for catalysis. ${ }^{9,10}$ In spite of this, only a few theoretical works ${ }^{11-14}$ have been devoted to study its surface properties (for a review on TiN and other nitrides see Ref. 15). In particular, we have recently reported an investigation on the relaxation of the (001) surface for several transitionmetal nitrides as well as the interaction of $\mathrm{Au}$ atoms with those surfaces using p-DFT calculations. ${ }^{16}$ In the present paper, as a part of our continuous effort to unravel the properties of such interfaces, we report on the classical MD simulations of the TiN (001) surface and gold clusters deposited on it (Sec. IV).

\section{THEORETICAL ASPECTS}

Let us assume two groups of atoms (A and B) inside a super cell. They are placed so that repeating the cell in the

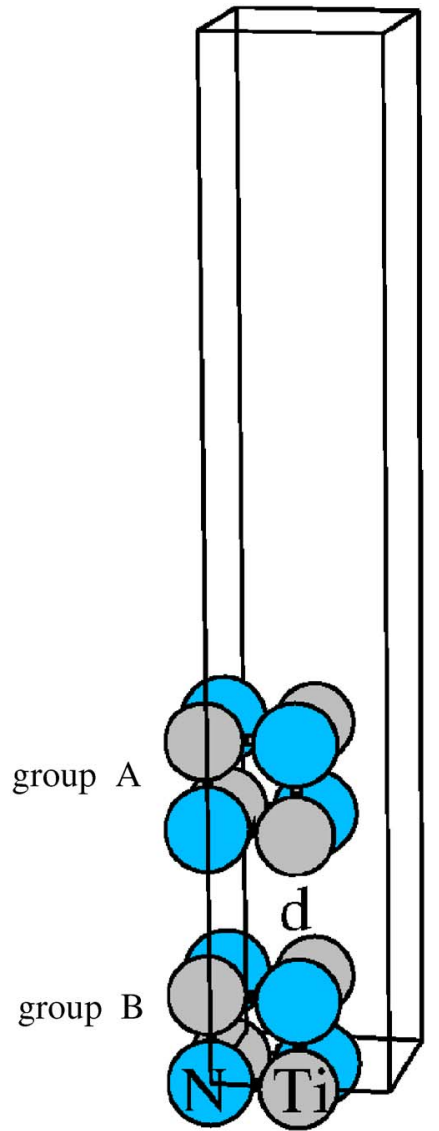

FIG. 1. Scheme of the supercell containing two groups of atoms (A and B) used to calculate the interaction energy from the DFT calculations. The vacuum space over group $\mathrm{A}$ is large enough to neglect the interaction with outer cell film.

three directions two films of A and B separated by $d(\AA)$ are created (Fig. 1). In addition, the model has an enough empty space on top of group A in order to minimize the interaction energy with other films obtained by repeating the cell in $z$ direction.

The idea of the method is to generate a set of pair potentials between atoms of groups A and B, so that when calculating the interaction energy between $\mathrm{A}$ and $\mathrm{B}$ films at distance $d$ (using cMD interaction potentials), $E_{\mathrm{MD}}^{\mathrm{int}(\mathrm{A}, \mathrm{B}, d)}$, we obtain the same result as using the p-DFT calculations $E_{\mathrm{p}-\mathrm{DFT}}^{\mathrm{int}(\mathrm{A}, \mathrm{B}, d)}$

$$
E_{p-\mathrm{DFT}}^{\mathrm{int}(\mathrm{A}, \mathrm{B}, d)} \approx E_{\mathrm{MD}}^{\mathrm{int}(\mathrm{A}, \mathrm{B}, d)} .
$$

The interaction energy $E_{\mathrm{p}-\mathrm{DFT}}^{\mathrm{int}(\mathrm{A}, \mathrm{B})}$ is computed by

$$
E_{p-\mathrm{DFT}}^{\mathrm{int}(\mathrm{A}, \mathrm{B}, d)}=E_{p \text {-DFT }}^{\mathrm{A}, \mathrm{B}, d}-E_{p-\mathrm{DFT}}^{\mathrm{A}}-E_{p \text {-DFT }}^{\mathrm{B}},
$$

where the first term of the right member is the energy of the cell including $\mathrm{A}$ and $\mathrm{B}$ atoms at distance $d$, while the next two terms correspond to the energies of isolated A and B groups. When the distance between the films is large enough $\left(d_{\infty}\right)$ the interaction between the two groups is mainly of long-range type.

On the other hand, the right member of (1) can be written in a similar way to Eq. (2),

$$
E_{\mathrm{MD}}^{\mathrm{int}(\mathrm{A}, \mathrm{B}, d)}=E_{\mathrm{MD}}^{\mathrm{A}, \mathrm{B}, d}-E_{\mathrm{MD}}^{\mathrm{A}}-E_{\mathrm{MD}}^{\mathrm{B}} .
$$


Taking into account that the interaction potential for MD is a sum of long-range and short-range terms, the interaction energy is

$E_{\mathrm{MD}}^{\mathrm{int}(\mathrm{A}, \mathrm{B}, d)}=\left(E_{\mathrm{MD}}^{\mathrm{long}(\mathrm{A}, \mathrm{B}, d)}-E_{\mathrm{MD}}^{\mathrm{long}(\mathrm{A})}-E_{\mathrm{MD}}^{\mathrm{long}(\mathrm{B})}\right)+E_{\mathrm{MD}}^{\prime \text { short }(\mathrm{A}, \mathrm{B}, d)}$,

where

$$
E_{\mathrm{MD}}^{\text {short}(\mathrm{A}, \mathrm{B}, d)}=E_{\mathrm{MD}}^{\text {short }(\mathrm{A}, \mathrm{B}, d)}-E_{\mathrm{MD}}^{\text {short(A)}}-E_{\mathrm{MD}}^{\text {short(B) }}
$$

is the contribution to the interaction energy only due to the short-range potentials between $\mathrm{A}$ and $\mathrm{B}$ atoms.

Increasing the value of $d$ causes $E_{\mathrm{MD}}^{\prime \text { short( }(\mathrm{A}, \mathrm{B}, d)}$ to become zero rapidly for any rational set of potentials chosen. The distance for which $E_{\mathrm{MD}}^{\text {short }(\mathrm{A}, \mathrm{B}, d)} \approx 0$ determines the value of the parameter $d_{\infty}$, mentioned above. The actual value of $d_{\infty}$ depends on the configuration adopted for atoms in both groups and on the cutoff distance $\left(r_{c}\right)$ proposed for the shortrange potentials. At the same time, $r_{c}$ is indirectly related to the form of the long-range potential, i.e., the charges assigned to the atoms. The key to this procedure is the correct selection of such charges in the groups $(q\{A\}, q\{B\})$ and the cutoff radius $r_{c}$ for each pair of atoms. In principle, expression (1) is always fulfilled for any set of charges, $q\{A\}, q\{B\}$, and $r_{c}$, but not always in all cases is a reasonable short distance $d_{\infty}$ between the groups found so that the short-range interaction vanishes.

Replacing Eqs. (4) and (2) in (1) we obtain

$$
\begin{aligned}
& E_{p-\mathrm{DFT}}^{\mathrm{A}, \mathrm{B}, d}-E_{p-\mathrm{DFT}}^{\mathrm{A}}-E_{p-\mathrm{DFT}}^{\mathrm{B}}-\left(E_{\mathrm{MD}}^{\mathrm{long}(\mathrm{A}, \mathrm{B}, d)}-E_{\mathrm{MD}}^{\mathrm{long}(\mathrm{A})}-E_{\mathrm{MD}}^{\mathrm{long}(\mathrm{B})}\right) \\
& \quad=E_{\mathrm{MD}}^{\text {short}(\mathrm{A}, \mathrm{B}, d)}
\end{aligned}
$$

and a similar equation for $d_{\infty}$,

$$
\begin{aligned}
& E_{p \text {-DFT }}^{\mathrm{A}, \mathrm{B}, d_{\infty}}-E_{p \text {-DFT }}^{\mathrm{A}}-E_{p \text {-DFT }}^{\mathrm{B}}-\left(E_{\mathrm{MD}}^{\operatorname{long}\left(\mathrm{A}, \mathrm{B}, d_{\infty}\right)}-E_{\mathrm{MD}}^{\text {long(A) }}-E_{\mathrm{MD}}^{\text {long(B) }}\right) \\
& \quad=E_{\mathrm{MD}}^{\prime \text { short }\left(\mathrm{A}, \mathrm{B}, d_{\infty}\right)} \approx 0 .
\end{aligned}
$$

With the aim of avoiding the calculation of $E_{\mathrm{p}-\mathrm{DFT}}^{\mathrm{A}}$, $E_{\mathrm{p}-\mathrm{DFT}}^{\mathrm{B}}, E_{\mathrm{MD}}^{\text {long(A) }}$, and $E_{\mathrm{MD}}^{\text {long(B) }}, \mathrm{Eq} .(7)$ is subtracted from the set of equations (6). Following this, we also assure that the set of the pairwise potentials vanishes at $r_{c}$. Finally, we obtain the set of equations (as many as points $d_{i}$ chosen):

$E_{p \text {-DFT }}^{\mathrm{A}, \mathrm{B}, d}-E_{p \text {-DFT }}^{\mathrm{A}, \mathrm{D}, d_{\infty}}-\left(E_{\mathrm{MD}}^{\mathrm{long}(\mathrm{A}, \mathrm{B}, d)}-E_{\mathrm{MD}}^{\mathrm{long}\left(\mathrm{A}, \mathrm{B}, d_{\infty}\right)}\right)=E_{\mathrm{MD}}^{\prime \text { short(A,B,d) }}$.

In Eq. (8) the terms related to the long-range interaction are easily computed using the Ewald ${ }^{17}$ sum procedure once the atomic charges of the $\mathrm{A}$ and $\mathrm{B}$ groups have been assigned.

Let us assume that groups $\mathrm{A}$ and $\mathrm{B}$ are composed of different species of atoms. Then there are as many interaction potentials to obtain as the number of pair species $j k$ that can be formed. We will call these the pair potential $j k$ or $P_{j k}$. In addition to the cutoff radius for a given $j k$ pair, $r_{c}^{j k}$, let us define a minimum radius $\left(r_{\text {min }}\right)$, so that two species $j k$ cannot be closer than the distance $r_{\min }^{j k}$ for any type of calculation (p-DFT or cMD).

In principle, our task is to obtain a set of functions $P_{j k}(r)$ with $r_{\min }^{j k}<r<r_{c}^{j k}$. Because $P_{j k}(r)$ are continuous, we do not need necessarily to know the mathematical form of $P_{j k}(r)$, but the values of these functions at some points $r_{i}^{j k}$, where $r_{\min }^{j k}<r_{i}^{j k}<r_{c}^{j k}$, i.e., to change to the discrete space on $r$. Therefore, our task is to obtain $i=1,2, \ldots, N_{j k}$ components of the vector $P_{j k}$ for each $j k$ pair of species. The right-hand side of Eq. (8) can then be written as a linear combination of $P_{j k}^{i}$ as

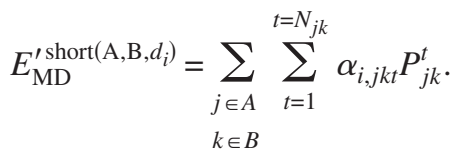

To solve this system we need as many equations (calculated configurations) as unknown short-range vector components, $N$ (with $N=\sum_{\substack{k \in B \\ j \in A}} N_{j k}$ ), and, in addition, the equations must be linearly independent. After solving the system of equations (9), the short-range contribution to the pairwise interaction potential is obtained.

In Eq. (9), the coefficients $\alpha_{i, j k t}$ represent the number of times that the term $P_{j k}^{t}$ appears in the right-hand side of Eq. (8). They are calculated by forming all the possible pairs of atoms between $\mathrm{A}$ and $\mathrm{B}$ groups, fulfilling the condition $r_{\text {min }}^{j k}$ $<r<r_{c}^{j k}$, taking into account not only the computational cell but also the neighbor cells.

\section{DETERMINATION OF INTERACTION POTENTIALS}

The first step of the simulations was to determine the force field governing the system behavior that, according to our scheme, and considering first the support, TiN, involves determination of three parameters $P_{\mathrm{TiTi}}, P_{\mathrm{TiN}}$, and $P_{\mathrm{NN}}$ for a set values of $r$ in the $r_{\min }, r_{c}$ range (here set to 1 and $5 \AA$, respectively). With this aim we used an initial supercell in which groups A and B (two subcells) are placed at a distance $d$, which fulfills the $r_{\min }^{j k}<r<r_{c}^{j k}$ condition. Because of periodic boundary conditions, groups A and B actually represent two slabs of TiN, and there is a vacuum in the supercell to prevent intercell interaction between slabs. As shown in Fig. 1 , groups $\mathrm{A}$ and $\mathrm{B}$ consisted of two Ti atoms and two $\mathrm{N}$ atoms arranged in slabs two-layer thick. In order to get a dense mesh the distance interval should be divided in, say, 1000 steps, which obviously would involve quite cumbersome computations. Instead of that we preferred to compute the energy at slab distances $d$ going from 1 to $5 \AA$, step of $0.1 \AA$ (41 geometries), and to perform then an interpolation. The energy of these 41 structures was estimated from the DFT calculations using the VASP 4.5 (Refs. 18 and 19) code and the generalized gradient approach (GGA) ${ }^{20}$ Full details on these calculations can be found in Ref. 13. After cubic spline interpolation a set of 1000 linearly independent equations of the form (9) was obtained. Since we have only a third of the equations required, we repeat the process for two more initial different configurations, obtaining a final set of 3000 equations that after solving lead to the 1000 components of vectors $P_{\mathrm{TiTi}}, P_{\mathrm{TiN}}$, and $P_{\mathrm{NN}}{ }^{5}$

To simulate the $\mathrm{Au} / \mathrm{TiN}$ interface we now need to estimate the interaction concerning $\mathrm{Au}$ atoms: $\mathrm{Au}-\mathrm{Au}, \mathrm{Au}-\mathrm{Ti}$, and $\mathrm{Au}-\mathrm{N}$ (assuming transferability of the $P_{\mathrm{TiTi}}, P_{\mathrm{TiN}}$, and $P_{\mathrm{NN}}$ vectors). As already reported, isolated gold atoms prefer to bind the TiN (001) surface on top of Ti atoms, with a small charge transfer towards the surface. However, when 


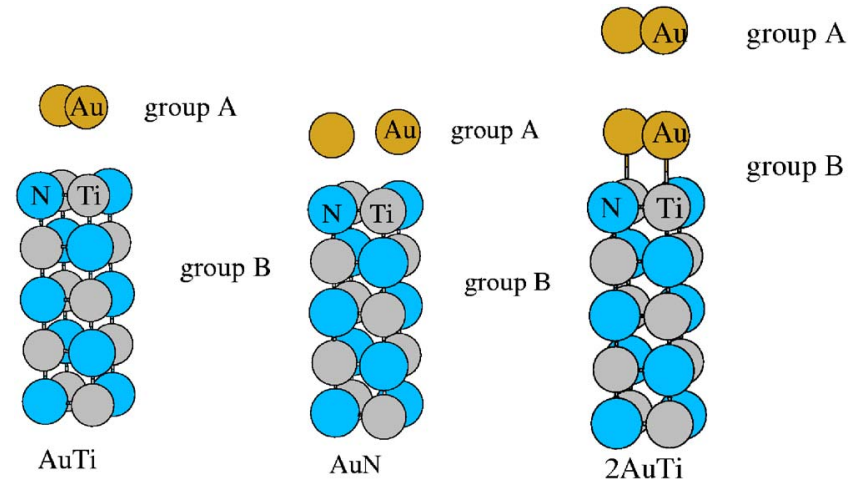

FIG. 2. The three initial configurations used to determine the pair potentials describing the interaction of Au with the surface.

$\mathrm{Au}_{2}$ dimers are supported, no such a transfer was observed. Similar behavior was also observed in the $\mathrm{Pd} / \alpha-\mathrm{Al}_{2} \mathrm{O}_{3}$ interface where adsorption of the metal cluster involved only a small polarization. ${ }^{6,21}$ These findings enabled us to limit the interaction potential of $\mathrm{Au}$ to the short-range contribution since it is obvious that in the interface higher coverage does occur. In a similar way to that exposed for TiN, three initial configurations were chosen to determine the pair interaction potentials $\mathrm{Au}-\mathrm{Ti}, \mathrm{Au}-\mathrm{N}$, and $\mathrm{Au}-\mathrm{Au}$ (Fig. 2). Here group A consists of two Au atoms in all cases and group B was either a slab of TiN (001) five-layer thick or two gold atoms deposited on such a slab. Gold atoms were placed on top of Ti and on top of N [Figs. 2(a) and 2(b)]. In Fig. 2(c) two gold atoms in group A interact with two gold atoms of group B, allowing to determine an interaction potential of the gold-adsorbed gold type. Following these configurations, the complete set of $E_{\mathrm{MD}}^{\text {short(A,B,d) }}$ values were obtained by variation of the distance $d$ so that $1.0 \AA<d<5.0 \AA$. The total pair interaction potential curves are shown in Fig. 3.

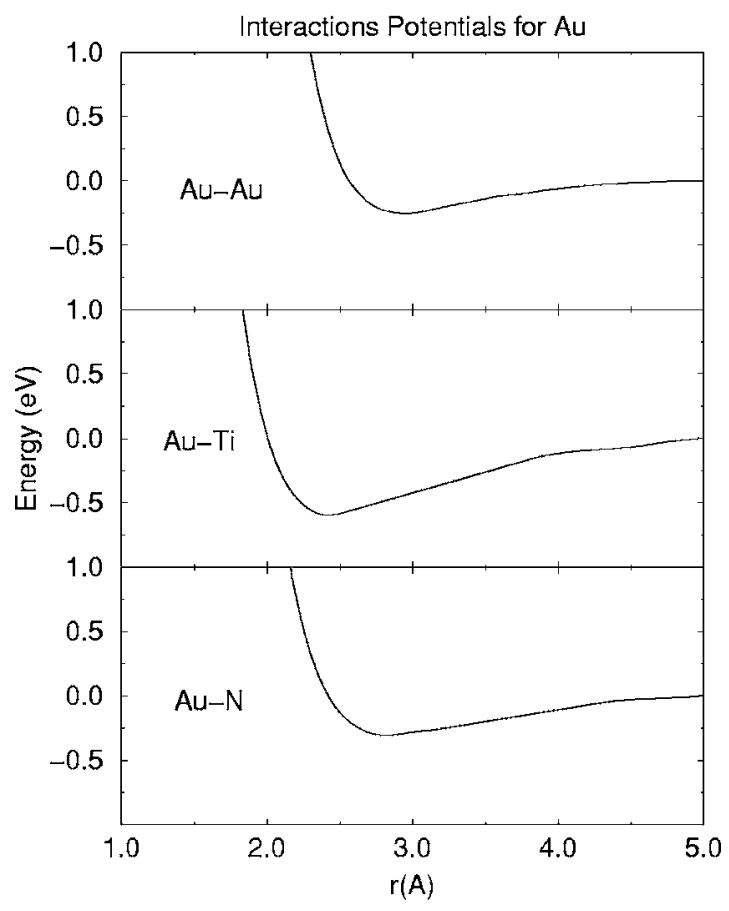

FIG. 3. Interaction potentials for $\mathrm{Au}-\mathrm{Au}, \mathrm{Au}-\mathrm{Ti}$, and $\mathrm{Au}-\mathrm{N}$ pairs.



FIG. 4. Radial distribution functions (RDFs) for Ti-N, N-N, and Ti-Ti pairs obtained from the MD simulations of TiN (001) surface.

\section{MD SIMULATIONS OF Au CLUSTERS DEPOSITED ON TiN (001) SURFACE}

The MD simulations were undertaken in the microcanonical ensemble using the DL_POLY computer code. ${ }^{22}$ The computational box consisted of a cube $34.032 \AA$ side with a TiN slab six-layer thick and an enough vacuum gap in the $\langle 001\rangle$ direction. In the simulations, the two lowest layers were kept frozen and 1024 atoms were moved simultaneously. The initial geometry of particles in the slab was set coherent with the cell parameter obtained from the DFT calculations: $a=4.254 \AA$ [experimental value $4.238 \AA$ (Ref. $23)$. On this surface, gold clusters of different sizes (200350 atoms) were deposited. The simulation proceeds by first assigning initial velocities to all atoms according to a Maxwell-Boltzmann distribution that depends on the temperature of the systems $(300 \mathrm{~K})$. From this starting point, Newton's equations of motion were solved by numeric integration using the leap-frog algorithm with a time step of $0.1 \mathrm{fs}^{24}$ The simulation schedule consisted of a thermalization run of $10 \mathrm{ps}$ followed by a simulation of $10 \mathrm{ps}$ without temperature control to ensure that the system reached equilibrium. After this stage, a simulation of 15 ps was carried out to collect data for statistics. The conservation of energy was better than 1 in 10000 .

The starting step of the simulations was to investigate the behavior of the TiN (001) isolated surface. The surface shows to be stable at that temperature, and the major features can be deduced from the radial distribution function (RDF) reproduced in Fig. 4. For the Ti-N pair the first band is large, with a peak at $2.1 \AA$, in agreement with the Ti-N interatomic distance of $2.127 \AA$ obtained from the DFT calculations. According to the fcc structure of TiN, this peak is followed by another at about $(a / 2) \sqrt{3} \AA$ due to the second-neighbor shell. 


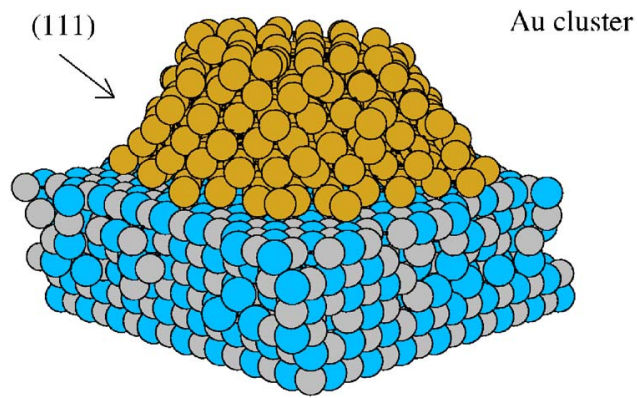

FIG. 5. Snapshot of the $\mathrm{Au}_{343}$ cluster deposited on the TiN (001) surface.

For Ti-Ti and N-N pairs, the most outstanding features are broad peaks slightly falling below $3 \AA$, i.e., somewhat lower than the theoretical distance of $(a / 2) \sqrt{2} \AA$, followed by bands about $4 \AA$, i.e., $a(\AA)$. Compared to the RDF obtained from bulk simulations, ${ }^{5}$ two main differences may be observed. Primarily, the first band of Ti-Ti and N-N pairs appear to be slightly shifted to shorter interatomic distances, suggesting that there is some contraction of the surface. Secondly, for these pairs, a shoulder in the second band is clearly observed. These shoulders are a consequence of the rippling relaxation of the surface, in which the outermost layers of nitrogen and titanium atoms are not in the same plane. The difference between the positions of these shoulders gives a rippling of $0.12 \AA$, in quite good agreement with that found from p-DFT calculations $(0.17 \AA) .{ }^{13}$ This result constitutes a test of the effectiveness of the pairwise interaction potentials obtained in the preceding sections.

In order to simulate the Au/TiN interface two gold clusters consisting of 216 and 343 atoms were added to the surface. (Since the results are practically identical, only the 343 atoms deposit will be reported.) A snapshot of the system after the simulations is reproduced in Fig. 5. The gold atoms closest to the surface are found on top of Ti atoms, in agreement with the largest $\mathrm{Au}-\mathrm{Ti}$ interaction. In the second layer, gold atoms are placed on the vertical above the outermost nitrogen atoms, bound to four first-layer gold atoms. The next layer is identical to the first one, according to an almost layer-by-layer $\mathrm{ABABAB}$ pattern. In fact, the structure of the $\mathrm{Au}$ atoms is like that of the titanium lattice in the nitride, corresponding to a compact packing fcc in agreement with the natural form that is determined experimentally for the bulk. This $(001)_{\mathrm{TiN}} \|(001)_{\mathrm{Au}}$ epitaxial relation is similar to that observed for gold clusters deposited on the $\mathrm{MgO}(001)$ surface from both high-resolution electron microscopy experiments and MD simulations. ${ }^{25-28}$ On the other hand, it is worth to note that there is a misfit between the Au and TiN lattices, being the $\mathrm{Au}$ cell parameter somewhat lower ( $a$ $=4.078$ and $4.254 \AA$, respectively). This misfit mainly affects the first layer of gold atoms, which is found to be slightly stressed, however, beyond this layer; the Au cluster gradually contracts to recover the metallic structure. Such an accommodation of the gold lattice has already been observed in the deposition of $\mathrm{Au}$ clusters on $\mathrm{MgO}$ (001) surfaces. ${ }^{28}$ The analysis of the Au cluster shows a morphology corresponding to an irregular polyhedron truncated along specific directions, exposing (111) and (001) facets.

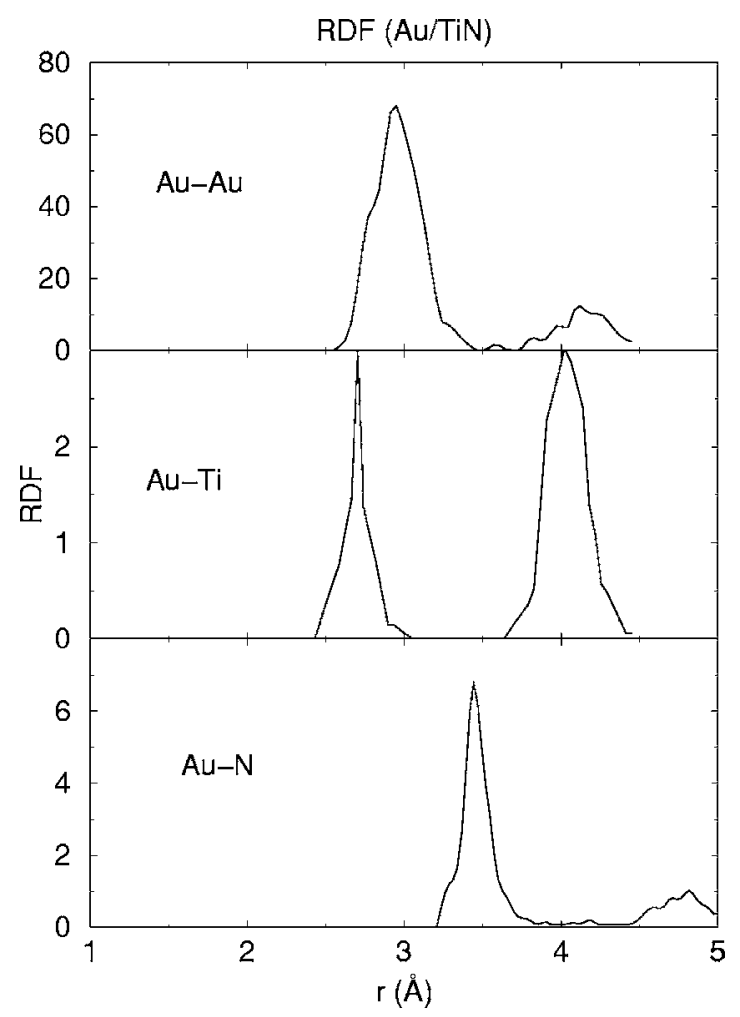

FIG. 6. Radial distribution functions (RDFs) for $\mathrm{Au}-\mathrm{Au}, \mathrm{Au}-\mathrm{Ti}$, and $\mathrm{Au}-\mathrm{N}$ pairs obtained from the MD simulations of Au cluster deposited on the TiN (001) surface.

The misfit between the TiN and Au lattices is also responsible for the wideness of the first band observed in the $\mathrm{Au}-\mathrm{Au} \mathrm{RDF}$ reported in Fig. 6. The main peak approximately appears at $2.9 \AA$, which is close to the experimental distance of $2.88 \AA .^{29}$ There is also another effect due to the undercoordination of Au atoms lying on the outermost layers of the cluster. These atoms tend to shorten the bond distances and give rise to the shoulder observed at lower distances. With respect to the other two pairs, the first peak of $\mathrm{Au}-\mathrm{Ti}$ $\mathrm{RDF}$ appears at $2.7 \AA$ and corresponds to $\mathrm{Au}-\mathrm{Ti}$ pairs of atoms directly bonded, i.e., the first layer of gold and outermost layer of the support. The second band, much more intense, also arises from these two layers, but involve $\mathrm{Au}-\mathrm{Ti}$ atoms not directly bound (one Au by each four Ti and vice versa). Finally, the most prominent feature of the $\mathrm{Au}-\mathrm{N} \mathrm{RDF}$ is the peak at $3.4 \AA$ originated from the first-layer $\mathrm{Au}$ atoms and outermost $\mathrm{N}$ atoms.

\section{SUMMARY AND CONCLUSIONS}

In this paper we report on a procedure that allows us to determine interaction pairwise potentials specifically tailored for the system under study. These ad hoc potentials are expressed as a sum of long- and short-range contributions and obtained, in our case, from the DFT calculations carried out under periodic boundary conditions. After solving a set of equations linearly independent, the potentials are obtained and directly used without further fitting to any analytical expression. Although the procedure is in principle of general purpose, our intention is to use it to simulate the surface properties of materials, and we report on an application of 
the complete procedure to TiN. The MD simulations of TiN (001) surface shows noticeable rumpling of titanium and nitrogen outermost layers, with a rippling relaxation parameter estimated from the radial distribution functions of $0.12 \AA$. This value is in reasonable agreement with that obtained using a full DFT approach, and supports the reliability of the potentials.

Simulations of a gold particle deposited on the TiN (001) surface shows that the transition-metal particle epitaxially grows on the surface, in such a way that the Au atoms are on top of surface Ti atoms. The gold particle shows fcc structure and mainly exposes the (111) and (001) facets. Because of the misfit of the $\mathrm{Au}$ and TiN lattices, the layer of gold atoms closest to the substrate is dilated, and gradually contracts towards the external region of the cluster to recover the metallic interatomic distance.

\section{ACKNOWLEDGMENTS}

The project was financially supported by the Spanish Ministerio de Ciencia y Tecnologia-Project No. MAT200501872 and the Ramon y Cajal program (awarded to N.C.H.) — and the Junta de Andalucía (Project No. FQM132).

${ }^{1}$ R. G. Parr and W. Yang, Density-Functional Theory of Atoms and Molecules (Oxford University Press, New York, 1989).

${ }^{2}$ M. C. Payne, M. P. Teter, D. C. Allan, T. A. Arias, and J. D. Joannopoulos, Rev. Mod. Phys. 64, 1045 (1992).

${ }^{3}$ R. Car and M. Parrinello, Phys. Rev. Lett. 55, 2471 (1985).
${ }^{4}$ M. P. Allen and D. J. Tildesley, Computer Simulation of Liquids (Claredon, Oxford 1987).

${ }^{5}$ N. C. Hernández and J. F. Sanz, Comput. Mater. Sci. (in press).

${ }^{6}$ N. C. Hernández and J. F. Sanz, J. Phys. Chem. B 105, 12111 (2001).

${ }^{7}$ M. Wittmer, J. Vac. Sci. Technol. A 3, 1797 (1985).

${ }^{8}$ L. E. Toth, Transition Metal Carbides and Nitrides (Academic, New York, 1971)

${ }^{9}$ S. Tsubota, M. Haruta, T. Kobayashi, A. Ueda, Y. Nakahara, and G. Poncelet Preparation of Catalysts V (Elsevier, New York, 1991), p. 695.

${ }^{10}$ M. A. Centeno and M. J. Lloret, Mater. Sci. Forum 383, 111 (2002).

${ }^{11}$ M. Marlo and V. Milman, Phys. Rev. B 62, 2899 (2000).

${ }^{12}$ J.-Q. Li, Y.-F. Zhang, S.-C. Xiang, and Y.-N. Chiu, J. Mol. Struct.: THEOCHEM 530, 209 (2000).

${ }^{13}$ N. C. Hernández, J. Graciani, and J. F. Sanz, Surf. Sci. 541, 217 (2003).

${ }^{14}$ C. V. Ciobanu, D. T. Tambe, and V. B. Shenoy, Surf. Sci. 582, 145 (2005).

${ }^{15}$ L. I. Johansson, Surf. Sci. Rep. 21, 177 (1995).

${ }^{16}$ N. C. Hernández and J. F. Sanz, Mater. Sci. Forum 383, 177 (2002).

${ }^{17}$ P. Ewald, Ann. Phys. (N.Y.) 64, 253 (1921).

${ }^{18}$ G. Kresse and J. Hafner, Phys. Rev. B 47, R558 (1993).

${ }^{19}$ G. Kresse and J. Furthmuller, Comput. Mater. Sci. 6, 15 (1996).

${ }^{20}$ J. Perdew, J. Chevary, S. Vosko, K. Jackson, M. Pederson, D. Singh, and D. Fiolhais, Phys. Rev. B 46, 6671 (1992).

${ }^{21}$ R. B. Gomes, F. Illas, N. C. Hernández, A. Márquez, and J. F. Sanz, Phys. Rev. B 65, 125414 (2002).

${ }^{22}$ W. Smith and T. R. Forester, J. Mol. Graphics 14, 136 (1996).

${ }^{23}$ N. Schoenberg, Acta Chem. Scand. 8, 213 (1954).

${ }^{24}$ L. Verlet, Phys. Rev. 159, 98 (1967).

${ }^{25}$ S. Giorgio, C. Chapon, C. R. Henry, G. Nihoul, and J. M. Penisson, Philos. Mag. A 64, 87 (1991).

${ }^{26}$ T. Kizuka, T. Kachi, and N. Tanaka, Z. Phys. D: At., Mol. Clusters 26, S58 (1993)

${ }^{27}$ T. Kizuka and N. Tanaka, Phys. Rev. B 56, R10079 (1997).

${ }^{28}$ B. Pauwels, G. Van Tendeloo, W. Bouwen, L. Theil Kuhn, P. Lievens, H. Lei, and M. Hou, Phys. Rev. B 62, 10383 (2000).

${ }^{29}$ A. Maeland and T. B. Flanagan, Can. J. Phys. 42, 2364 (1964). 